\title{
Nanoscale Nonlinear Optical Spectroscopy with Electron Beams
}

Andrea Konecna ${ }^{1}$, Valerio Di Giulio ${ }^{1}$, Vahagn Mkhitaryan ${ }^{1}$, Claus Ropers ${ }^{2}$ and F. Javier Garcia de Abajo ${ }^{1}$

${ }^{1}$ ICFO - The Institute of Photonic Sciences, Castelldefels, Catalonia, Spain, ${ }^{2}$ University of Gottingen, Göttingen, Niedersachsen, Germany

Electron energy loss spectroscopy (EELS) nowadays allows us to acquire information on excitations in nanostructures with high spatial and spectral resolution over a broad energy range covering vibrational, optical (valence-electron) and core-electron excitations $[1,2]$. The possibility of nanoscale mapping of optical response beyond the diffraction limit has made EELS one of the leading techniques in characterization of nanophotonic structures. However, EEL spectra are typically dominated by linear optical response, as the nonlinear contributions are negligible due to weak interaction between electrons and sample, which prevents the use of EELS in studying the nanoscale behavior of nonlinear optical fields.

The interaction between electrons and optical excitations in a sample can be substantially increased if the sample is excited by external optical pumping. Such a scenario is possible in photon-induced near field electron microscopy (PINEM), where we can reach scattering probabilities of order unity, and obtain multiple energy quanta exchanges between the electron probe and the optical field [3, 4]. Additionally, high light intensities can also trigger substantial nonlinearities in the sample response, which should be reflected in measured PINEM spectra.

In our work [5], we suggest PINEM as a suitable technique for nanoscale mapping of nonlinear optical response. In particular, we show that the interaction of electrons with nonlinear optical fields introduces asymmetries in otherwise symmetric PINEM spectra, which we demonstrate on several realistic examples. First we focus on studying second-harmonic (SH) response of spherical gold nanoparticles. We show that the evanescent $\mathrm{SH}$ field, which cannot be probed by conventional nonlinear optical spectroscopies, gives rise to substantially asymmetric electron spectra under achievable illumination intensities. We further calculate SH field in gold nanorods and show that significant asymmetries in the PINEM spectra can be achieved for particular beam positions when the linear-field coupling is reduced. We envision that PINEM performed with variable illumination frequency and intensity could become a powerful technique for characterization of nonlinear optical response with unsurpassed combination of spatial and spectral resolution [6].

References

[1] P. E. Batson, N. Dellby, and O. L. Krivanek, Nature 418, 617 (2002).

[2] O. L. Krivanek, T. C. Lovejoy, N. Dellby, T. Aoki, R. W. Carpenter, P. Rez, E. Soignard, J. Zhu, P. E. Batson, M. J. Lagos, et al., Nature 514, 209 (2014).

[3] B. Barwick, D. J. Flannigan, and A. H. Zewail, Nature 462, 902 (2009).

[4] A. Feist, K. E. Echternkamp, J. Schauss, S. V. Yalunin, S. Schäfer, and C. Ropers, Nature 521, 200 (2015).

[5] A. Konečná, V. Di Giulio, V. Mkhitaryan, C. Ropers, and F. J. García de Abajo, arXiv 1912.01539 (2019).

[6] Supported by ERC (Advanced Grant 789104-eNANO), the Spanish MINECO (MAT2017-88492-R and SEV2015- 0522), the Catalan CERCA Program, Fundació Privada Cellex, and the Deutsche Forschungsgemeinschaft (SFB 1073, project A05). V.D.G. acknowledges support from the EU through a Marie Skłodowska-Curie grant (COFUND-DP, H2020-MSCA-COFUND-2014, GA n 665884). 